\title{
APPLICATION OF SENSITIVITY ANALYSIS TO THE CORRECTION OF STATIC CHARACTERISTICS OF A PHASE ANGLE MODULATOR
}

\section{Ryszard Sroka}

AGH University of Science and Technology, Department of Measurement and Instrumentation, Al. Mickiewicza 30, 30-059 Cracow, Poland (凹rysieks@agh.edu.pl, +48 126173972 )

\begin{abstract}
The paper presents definitions and relative measures of the system sensitivity and sensitivity of its errors. The model of a real system and model of an ideal measuring system were introduced. It allows to determine the errors of the system. The paper presents also how to use the error sensitivity analysis carried out on the models of the measuring system to the correction of the nonlinearity error of its static characteristic. The corrective function is determined as a relation between the input variable of the tested system and its chosen parameter. The use of the proposed method has been presented on the example of a phase angle modulator. The obtained results have been compared with the results of analytic calculations. The idea of a phase angle modulator is also presented.
\end{abstract}

Keywords: phase modulator, sensitivity analysis, static characteristics, methods of correction.

(C) 2011 Polish Academy of Sciences. All rights reserved

\section{Introduction}

In the case of measurement systems, linearity of static characteristics is one of the most important parameters. Therefore measurement systems (sensors, transducers) are designed and constructed in such a way that their nonlinearity errors would be as small as possible. Often (particularly in case of new-generation sensors) the manufacturing technology used causes that obtaining good linearity in a wide range of input signals is very difficult (e.g. piezoresistive sensors, semiconductor temperature or pressure transducers, etc.). It enforces using, even at this stage of manufacturing, different types of compensation of influencing factors (e.g. temperature) and correcting systems for static characteristics.

Methods of correction of measurement transducer characteristics can be divided into two groups. The first one is using analog correction systems, often incorporated in a sensor or a transducer. The other one is using a digital system (often with a microprocessor) and a proper algorithm to eliminate the influence of unwanted factors or nonlinearity [1]. Integrated sensors employ both types of correction. However, both the first and the second method require previous knowledge of effects of the influence of external factors and nonlinearity in order to determine corrective functions that can be realized by an analog system or an algorithm in the digital equalizer.

Therefore searching for new methods of correction is extremely important, and also for tools that would enable determination of corrective functions which facilitate minimization of uncertainty of the information obtained at the outputs of the measurement systems $[2,3,4,5$, $6,7,8,9,10,11]$.

This paper suggests the use of sensitivity analysis as a tool for specification of corrective functions in measuring transducers. The proposed method enables specification of corrective functions also in case of cooperation of several systems, any of which can have own nonlinear characteristics. 
It is realized on the basis of models of the corrected systems. In models described with not too-much-complicated mathematical formulas it is possible to search for corrective functions by means of analytical calculation methods. In case of high-complexity models with nonlinearities, discontinuities, etc., sensitivity analysis realized by means of simulation methods can be the only effective and efficient tool for this purpose, which is undoubtedly an advantage of the proposed method.

The idea of this method and its use in specifying corrective functions have been presented on the example of a phase angle modulator. The results obtained have been compared with the results of analytic calculations.

\section{Sensitivity analysis and its measures}

The work [12] defines sensitivity analysis as a method purposed for examination of relationships between data (information, signals) at the inputs and the outputs of the considered systems.

Sensitivity of complex measuring devices is a broader term. It encompasses not only the influence of the input signals but also the influence of variability of technical parameters of the devices and external factors on their output signals and errors.

In the case of measurement systems, the purpose of carrying out the sensitivity analysis is double. In reference to the measured signals (input), the aim is usually maximizing sensitivity. In reference to both system parameters and interfering signals, the aim is minimizing the sensitivity of the system to those factors.

Experimental specification of sensitivity of complex devices would require many structural changes and laboratory measurements, therefore it would be time-consuming and expensive. As results from literature, in the scope of sensitivity tests of control systems, electric circuits as well as electronic systems, simulation methods are most effective [13, 14, 15]. The simulation methods need a model which should not be a summary of all the knowledge concerning the considered process or system, but it should represent only the part of knowledge that is important with reference to the purpose of the model. The notion of the model is understood in this case as complex mathematical representations determining relationships between variables (signals) and parameters of systems or devices.

The fundamental and necessary condition for using models is their credibility, understood as sufficiently good representation (with a sufficiently small error) of all properties of the considered object or phenomenon important for the user [12, 16, 17].

In this paper local sensitivity methods are used focusing on examination of local (in a selected point of the space of parameters) influence of changes of model parameters on its output. It is assumed that the model is continuous in the selected point and that the inputoutput relation in the area of slight changes of a selected parameter is linear [18].

A very important term related with sensitivity analysis is the so-called measure of sensitivity. It can be relative, absolute or weighted, and its form depends on whether the model is deterministic or stochastic. Absolute measures are not very useful, though (so they are not used quite often), as the obtained results do not let compare the influence of different parameters of the model on its output $[13,14]$. A more often applied solution is using relative measures [12]. The simplest relative measure for models described by differential equations is given by the formula:

$$
s_{i}=\frac{p_{i}}{y} \frac{\partial y}{\partial p_{i}}
$$

where: $y$ - model output, $p_{i}-$ nominal value of the $i$-th parameter in the model, $s_{i}-$ sensitivity of the model to the $i$-th parameter. 
But metrology assumes specification of sensitivity as a number, therefore sensitivity measures are usually given as the norm $S_{i}=\left\|s_{i}\right\|$ of the formula obtained in the differentiation process.

Contemporary measuring systems are usually very complex structures controlled according to a specific algorithm and containing elements of processing directly measured input signals such as programmable or hardware processors. For such systems constructing differentiable models would require far reaching simplifications, which is extremely difficult or even impossible along with the credibility requirement [13]. Such models very often are not analytically differentiable, then it is impossible to determine sensitivity by means of methods of differentiation of models.

For this reason in the paper a new method of sensitivity measures calculation is used, proposed in [13].

In case of measuring systems, an issue equally important as sensitivity of system outputs is sensitivity of errors to changes of system parameters. Such analysis can enable optimal selection of the model structure, system solutions and values of the parameters for given conditions of measurement and influencing factors [13]. For determining the sensitivity of the system output and sensitivity of its errors, the model $R$ of the analyzed real system and the definitional model $R_{\text {def }}$ (model of an ideal system) are used. They are included as representations from the sets of functions of input variables and parameters in the sets of output variables with the following form:

$$
\begin{gathered}
y=R(u, p) \\
y_{\text {def }}=R_{\text {def }}(u)
\end{gathered}
$$

whereas errors of the real system can be specified in the following way:

$$
Q(u, p)=J\left(y-y_{d e f}\right)
$$

where: $u$ - vector of input variables, $y$ - vector of output variables of the real system model, $y_{d e f}$ - vector of output variables of the definitional model, $p$ - vector of the system parameters, $R$ - model of the real system, $R_{\text {def }}$ - model of the definitional system, $Q$ - error of the system, $J(\bullet)$ - functional determining the method of error calculation.

It has been assumed that it is possible to specify the definitional model that represents an ideal method of obtaining measurement results, independent of any parameters. That model is introduced due to the necessity of correct determination of errors of the real system model. Such ideal systems do not exist in reality and can only be approximated by systems composed of standard devices. Whereas models of such systems can be built and serve as reference models. For example in the case of static characteristics of measuring systems, the model of a perfect system can be written as a linear function $y=k \cdot u$ (where $k$ is constant).

Then the measure of output sensitivity can be described as a relative measure, which enables easy comparison of influence of different parameters, and its size can be expressed as $[\% / \%]:$

$$
S_{y}=\frac{\|R(u+\Delta u, p+\Delta p)-R(u+\Delta u, p)\|}{\|R(u+\Delta u, p)\| \frac{\|\Delta p\|}{\|p\|}}
$$

and sensitivity of error in a normalized form can be defined in the following way [13]: 


$$
S_{\varepsilon}=\frac{\left\|R(u+\Delta u, p+\Delta p)-R_{\text {def }}\right\|-\left\|R(u+\Delta u, p)-R_{\text {def }}\right\|}{\left\|R_{\text {def }}\right\| \frac{\|\Delta p\|}{\|p\|}}
$$

The form (6) of the error sensitivity measure enables comparison of those sensitivities for different output variables and different parameters, because it expresses the relative variability of errors caused by relative variability of parameters.

Formula (6) can be rewritten as:

$$
\begin{gathered}
\Delta y_{1}=\left\|R(u+\Delta u, p)-R_{\text {def }}\right\| \\
\Delta y_{2}=\left\|R(u+\Delta u, p+\Delta p)-R_{\text {def }}\right\| \\
\Delta y=\left|\Delta y_{2}-\Delta y_{1}\right| \\
c=\left\|R_{\text {def }}\right\| \frac{\|\Delta p\|}{\|p\|}=\text { const } \text { for } u=\text { const } \\
S_{\varepsilon}=\frac{\Delta y}{c}
\end{gathered}
$$

Fig. 1 presents model outputs of a hypothetic definitional and real system versus chosen model parameter $p$, for constant value of input signal $u$. The definitional model is independent of model parameters, then its output $y_{\text {def }}$ is constant. Output $y$ of the model $R$ of a real system is sensitive to parameter $p$ (for simplicity we take into consideration a linear relation). For $p=p_{1}$ in Fig. 1 distances are presented between outputs of real and ideal models: $\Delta y_{1}, \Delta y_{2}$ and $\Delta y$ according to (7), (8) and (9). Formula (11) allows to calculate error sensitivity. For successive values of parameter $p$ the sensitivity is constant, because of the linear relation of $y$ versus $p$. For $p=p_{2}$ we have $\Delta y_{1}=\Delta y_{2}$ and in consequence $\Delta y=0$. It means that according to (11) error sensitivity is equal to zero (see Fig. 1). A "ditch"appears on the sensitivity characteristics. Figure 1 shows that for the verge of the ditch (point 1) we have a nonlinearity error equal to zero $\left(y=y_{d e f}\right)$. Then it is possible to calculate the error sensitivity of the system for other values of the input signal and to determine next points on the verge of the ditch. So we are able to find the relationship between the input signal and parameter $p$ (for which the verge of ditch appears), which can be used for correction of nonlinearity of the static characteristics of measuring systems.

The paper presents the proposed method of correction on the example of a phase angle modulator.

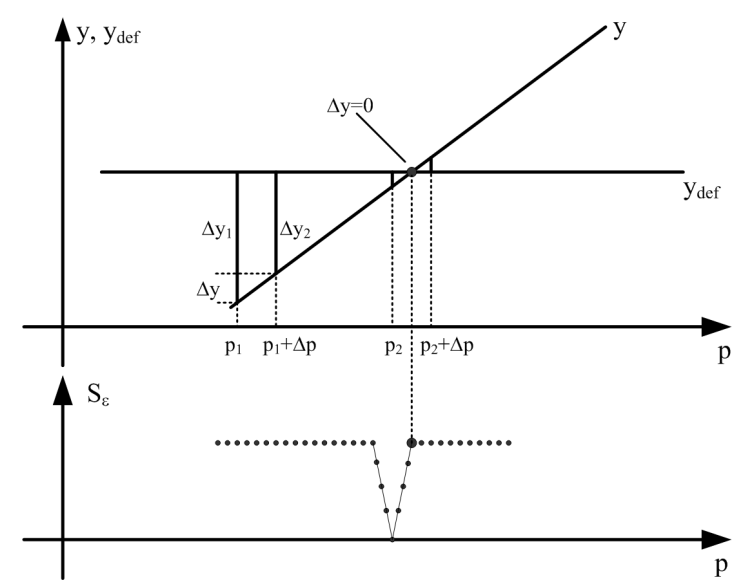

Fig. 1. Model outputs versus the analyzed model parameter and corresponding error sensitivity. 
Sensitivity tests should be carried out on models whose parameters have been preliminarily selected so that the errors would be minimal. After finding a set of values of parameters minimizing errors, measures of error sensitivity to individual parameters in the neighbourhood of their nominal values should be examined. During the test of influence of a selected parameter, all the others should have their nominal values.

\section{Idea of a phase angle modulator}

Modulation of phase (phase angle between two signals with the same frequency) is currently a rarely encountered method of processing signals in measuring systems. Despite of numerous advantages of this modulation method (small sensitivity to supply voltage, easy phase demodulation, etc.), phase modulators used until now were characterized by low range of phase deviation (around $\pm 0.2 \mathrm{rad}$ ) [19]. Nonlinearity errors of the static characteristic of a classic PM modulator (modulator with reference signal $u_{r}$ out of phase by the angle of $\pi / 2$ [rad] in relation to the supply voltage $\left.u_{c}[19]\right)$ reaching $1 \%$. The idea of such a modulator is presented in Fig. 2. Such a modulator can cooperate with parametric transducers of nonelectrical quantities (strain gauge, inductive or capacitive sensors) operating in a bridge or differential systems with output voltage $u_{o}$.

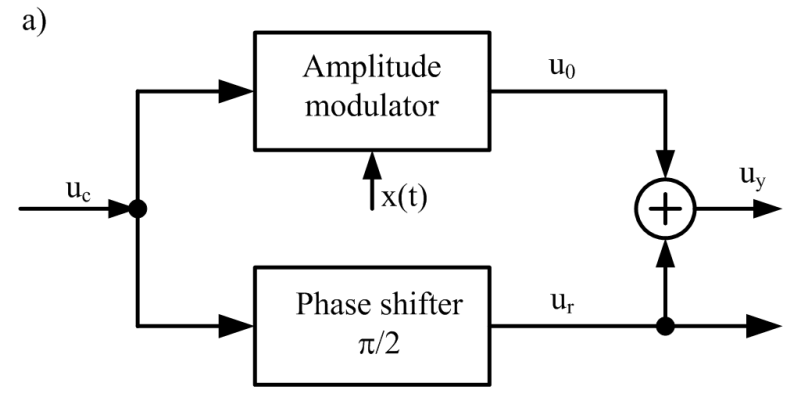

b)

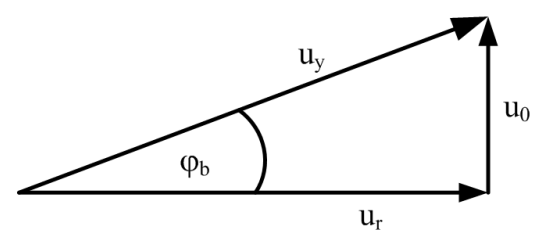

Fig. 2. PM modulator with reference signal shifted by angle $\pi / 2$ : a) block diagram, b) vector diagram.

In effect we receive a strongly nonlinear relation between the amplitude of signal $u_{o}$ and the output angle $\varphi_{b}$, given by the following formula:

$$
\varphi_{b}=\operatorname{arctg} \frac{\left|U_{o}\right|}{\left|U_{r}\right|}
$$

where: $U_{o}, U_{r^{-}}$signal amplitudes, respectively: $u_{o}, u_{r}$.

A new method of phase modulation characterized by a wide range of angle deviation (around $\pm 1.57 \mathrm{rad}$ ) and nonlinearity errors not exceeding $0.9 \%$ has been proposed in [14]. The considered system is a modification of a classic PM modulator. The modification consists in geometric summing of signal $u_{o}$ modulated in amplitude with reference signal $u_{r}$ with constant amplitude. In this solution the reference signal $u_{r}$ is out of phase in relation to the supply voltage by the angle $\varphi_{r}$, different from $\pi / 2$ [rad] [15]. The idea of the modified modulator is presented in Fig. 3. 


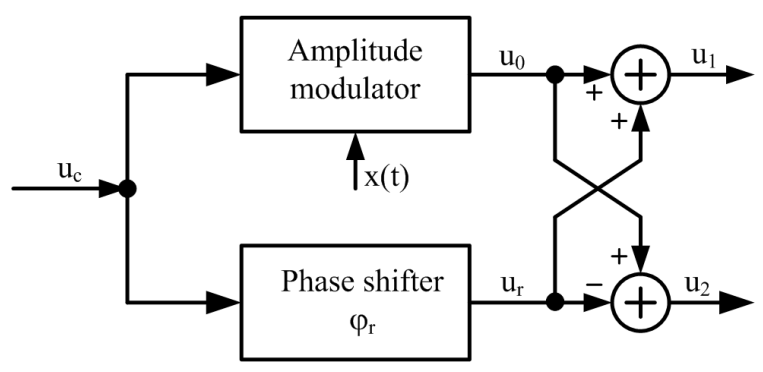

Fig. 3. Modified PM modulator with reference signal shifted by an angle different from $\pi / 2$.

Such modification enables obtaining approximately a ten times wider range of phase deviation with comparable (below 1\%) errors of nonlinearity of static characteristics of the modulator. The angle that is the result of the modulation is described as:

$$
\varphi(\alpha)=\left\{\begin{array}{ccc}
\operatorname{arctg} \frac{2 \cdot \alpha \cdot \sin \varphi_{r}}{1-\alpha^{2}} & \text { for } & |\alpha| \leq 1 \\
\pi+\operatorname{arctg} \frac{2 \cdot \alpha \cdot \sin \varphi_{r}}{1-\alpha^{2}} & \text { for } & \alpha>1 \\
-\pi+\operatorname{arctg} \frac{2 \cdot \alpha \cdot \sin \varphi_{r}}{1-\alpha^{2}} & \text { for } & \alpha<-1
\end{array}\right.
$$

where: $\alpha=U_{o} / U_{r}$.

Formula (13) is a static model of the modulator system. This model has been constructed with the assumption that the difference of phases of the supply voltage $u_{c}$ and modulated voltage $u_{o}$ is near zero (variable $\alpha$ is positive) or near $180^{\circ}$ (in this case variable $\alpha$ is negative). The above model has two parameters. They are: voltage amplitude $u_{r}$ and voltage phase $\varphi_{r}$. Due to parametric optimization of model (13), the nonlinearity error has been minimized, described by the following equation:

$$
\delta=\frac{\varphi(\alpha)-k_{\varphi} \cdot \alpha}{\varphi_{\max }} \cdot 100 \%
$$

Optimization has been carried out with the assumption that $U_{o}$ can belong to interval $\langle 0,1\rangle$ whereas signal $u_{o}$ has the possibility of a $180^{\circ}$ phase shift, and $U_{r}=1 \mathrm{~V}$. Angle $\varphi_{r}$ and $k_{\varphi}$ (factor of inclination of a straight line best approximating the modulation characteristics (definitional model $R_{d e f}$ ), by means of the LMS method) has been matched. In the result of optimization, in case $\left|\alpha_{\max }\right|=1$, have been obtained: $\varphi_{r}=0.8551 \mathrm{rad}$ and $k_{\varphi}=1.59$ with maximum nonlinearity error $\delta=0.88 \%$ and $\varphi_{\max }=1.585 \mathrm{rad}$. The nonlinearity error of the modulator after optimization is presented in Fig. 4.

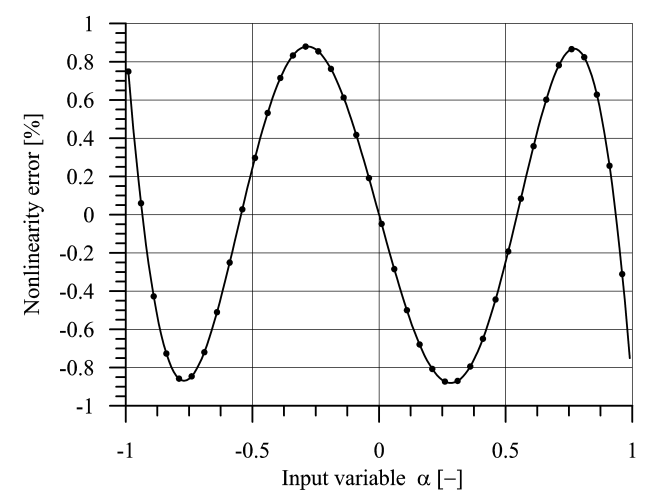

Fig. 4. Nonlinearity error of the static characteristic of the modified modulator after optimization. 
Beside the input variable range assumed for the optimization, nonlinearity errors of the static characteristics grow rapidly. The obtained values of the parameters have been assumed as optimal for the considered modulator model. Despite the wide range of deviation of the output angle, nonlinearity error of the static characteristics of the considered modulator model, reaching a value close to $0.9 \%$, in the context of the requirements set for contemporary measuring systems is not acceptable. Then a correction method should be found.

A tool that enables the development of corrective methods to facilitate further improvement of accuracy of such a modulator is presented before an analysis of error sensitivity. It allow to find the relationship between the input variable and a chosen model parameter, which will be retuned. Assuming that the measuring system consists of a sensor (amplitude modulator) and a signal conditioning system (phase modulator in this case), the structure of the system with the correction is presented in Fig. 5.

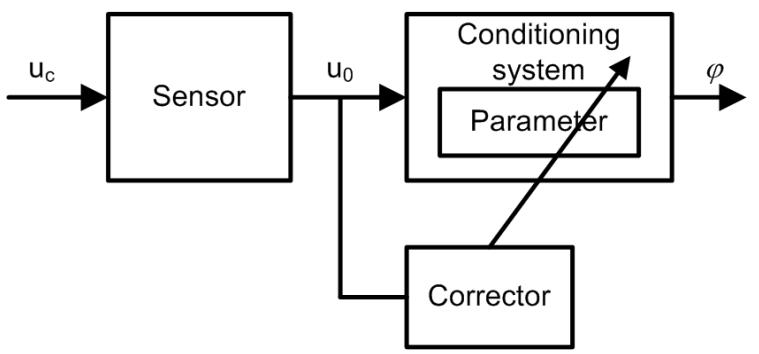

Fig. 5. Proposed structure of the system with the corrector.

In case of the considered modulator, the analysis of error sensitivity has been carried out due to the influence of both its parameters, i.e.: $U_{r}$ and $\varphi_{r}$. During the analysis of influence of one of the parameters, the other one had the specified value equal to the optimal. Parameter deviation has been assumed as $1 \%$ of its current value. Due to nonlinearity of the static characteristic of the modulator, sensitivity in each point can be different. In reference to that, analyses have been carried out for different points (different values of input variable) of that characteristic. The error sensitivity of the modulator to parameter $\varphi_{r}$ for $\left|\alpha_{\max }\right|=1$ has been presented in Fig. 6. In the presented characteristics of error sensitivity the abovementioned specific ,ditch” can be noticed.

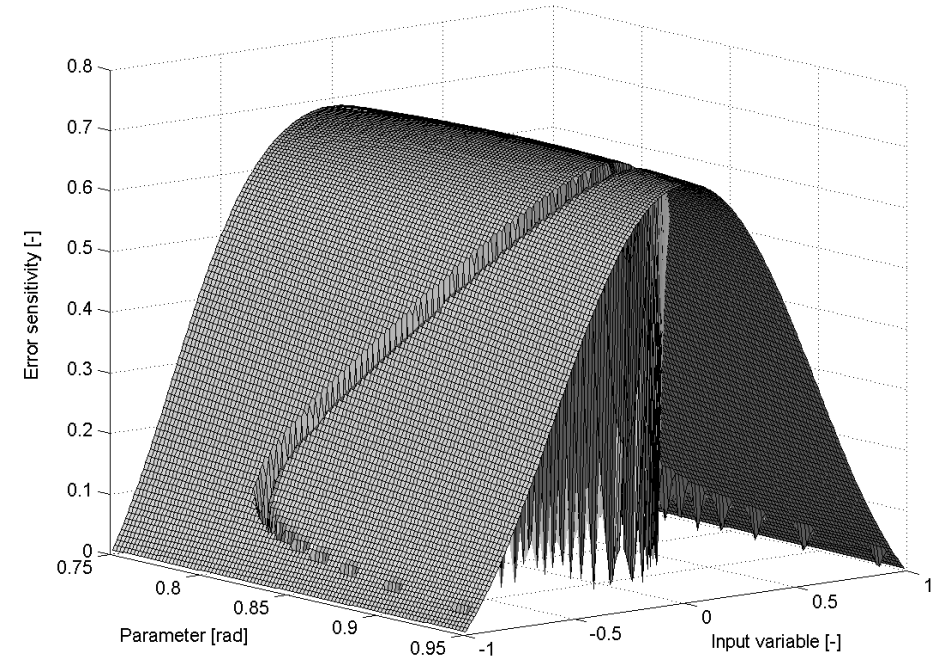

Fig. 6. Error sensitivity of the modulator to parameter $\varphi_{r}$ for input variable $\alpha \in(-1,1)$. 
Projecting the verge of the ditch on the plane parameter-input variable, a curve is obtained. The equation describing this curve links the current operating point on the static characteristics with the value of the model parameter providing zero value of the nonlinearity error of this characteristic. Thereby, an equation that is supposed to be realized by a possible corrective system changing the value of the parameter of the system depending on the current value of the input signal is obtained.

It has been assumed that the required characteristics of processing is supposed to be a linear function, with processing factor $k$, in the following form:

$$
y_{\text {def }}=k \cdot \alpha
$$

The equation (15) is a definitional model of the considered measurement system. In case of the considered phase modulator that has a relatively simple model, analytical specification of the corrective function is also possible. To do this, there should be selected a parameter that would be subject to the correction and the following equation should be solved:

$$
k \cdot \alpha=\operatorname{arctg} \frac{2 \cdot \alpha \cdot \sin \varphi_{r}}{1-\alpha^{2}}
$$

specifying this parameter as a function of all variables and the remaining parameters of the system. This method enables correction of a global error of nonlinearity of the whole measurement chain, from the input to the point where the correction is made. form:

For parameter $\varphi_{r}$, according to (16), a corrective function can be specified in the following

$$
\varphi_{r}=\arcsin \frac{\left(1-\alpha^{2}\right) \cdot \operatorname{tg}(k \cdot \alpha)}{2 \cdot \alpha}
$$

In Fig. 7 the curve described by equation (17) and the projection of the verge of the ditch from Fig. 6 on the plane parameter-input variable have been put together. We can see very good conformity of results of both methods.

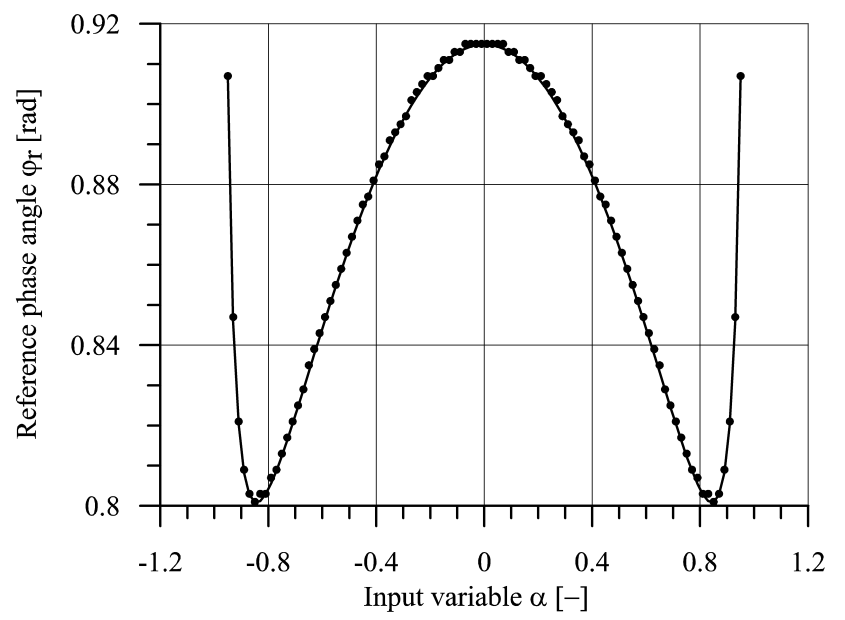

Fig. 7. Comparison of the corrective curve determined analytically (solid line) and obtained on the basis of the analysis of errors sensitivity (points), for parameter $\varphi_{r}$.

Application of a corrector that realizes a corrective function specified in this way results in obtaining the ideally linear static characteristics of the modulator system (see Fig. 8). 


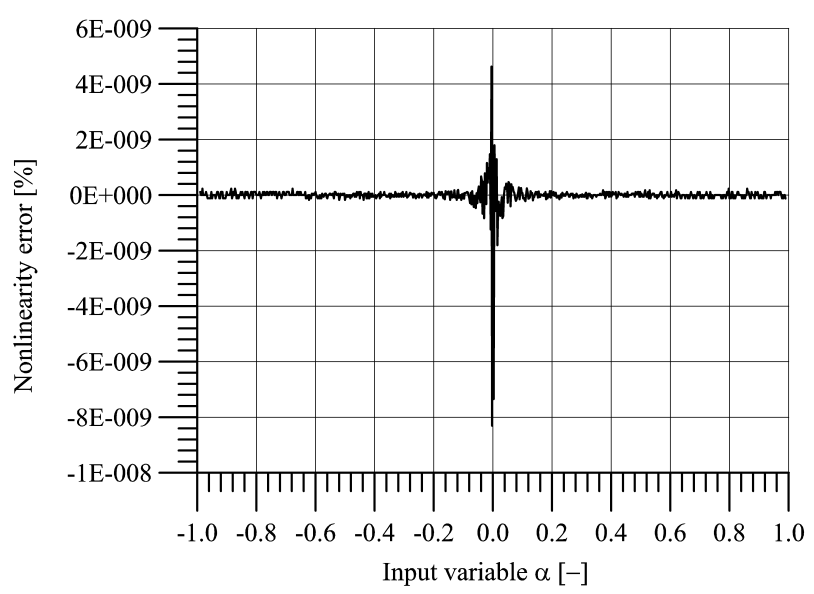

Fig. 8. Nonlinearity error of the modulator after correction.

A similar analysis has been carried out for parameter $U_{r}$. The error sensitivity to this parameter for the input variable from the interval $\langle-1,1\rangle$ has been presented in Fig. 9. A "ditch" also appears on the specified characteristics, which indicates that this parameter can be also used for correction of the nonlinearity error of the static characteristics.

Also in this case a solution for the problem of correction has been determined analytically, obtaining the formula:

$$
U_{r}=\frac{-U_{o} \operatorname{tg}\left(k \cdot U_{o}\right)}{\sin \varphi_{r} \pm \sqrt{\left(\sin \varphi_{r}\right)^{2}+\left[\operatorname{tg}\left(k \cdot U_{o}\right)\right]^{2}}}
$$

The result obtained in this way has been compared to the projection of the ditch of the sensitivity characteristics (see Fig. 10). Also in this case the conformity is very good. The obtained interval of retuning of the corrective parameter is $0.95 \div 1.01 \mathrm{~V}$.

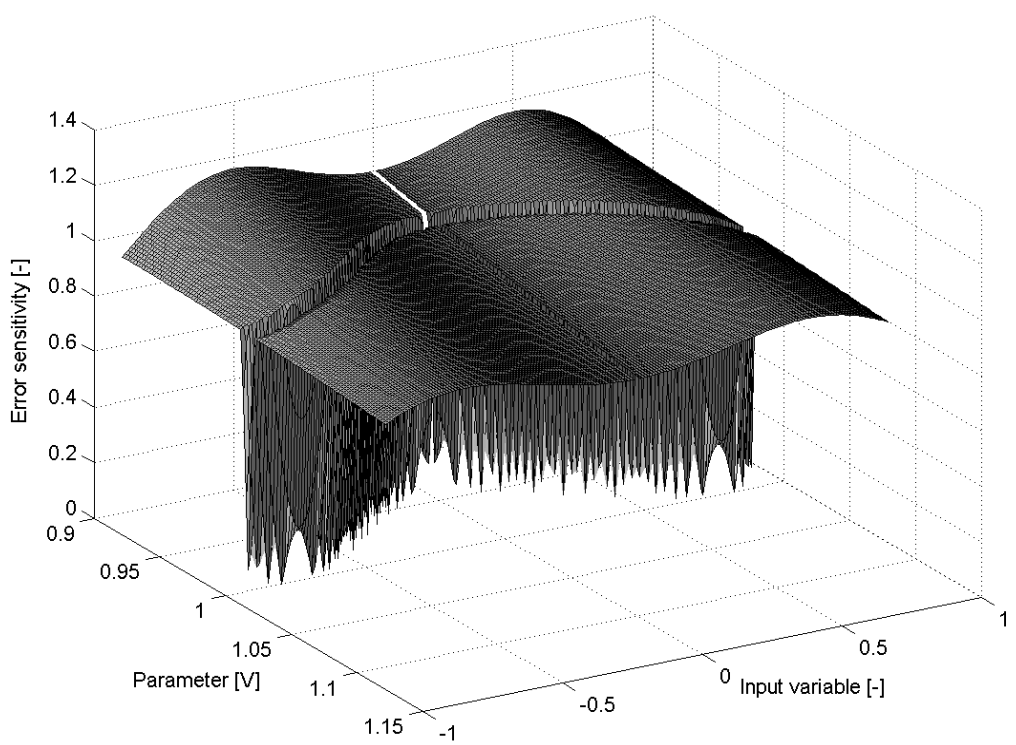

Fig. 9. The error sensitivity of the modulator to parameter $U_{r}$ for input value $\alpha \in(-1,1)$. 


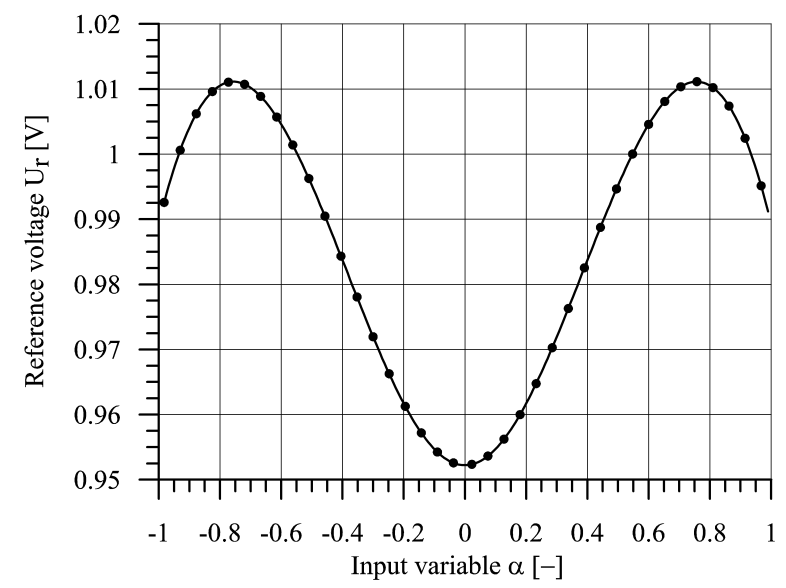

Fig. 10. Comparison of the corrective curve determined analytically (solid line) and obtained on the basis of the analysis of error sensitivity (points), for parameter $U_{r}$.

\section{Conclusions}

The paper presents the new method of specification of correction functions. It is destined for nonlinearity correction of static characteristics of measuring systems. This method uses the sensitivity analysis and in particular a quite rarely applied error sensitivity analysis. The results of using corrective functions specified in this way have been compared with the results obtained in analytical calculations, obtaining very good conformity. Nevertheless, the huge advantage of the proposed method in case of hardly differentiable models needs to be emphasised. The new correction method was presented on the example of a phase angle modulator. The basic condition of the proposed method is possession of a very credible model of the considered system, and carrying out of the sensitivity analysis on models optimized for the specified changes of the input value.

\section{References}

[1] Bryzek, J. (2004). Evolution of MEMS-IC Integration Technology. Special Issue of IEEE Measurement and Control Magazine.

[2] Kelly, G. (1999). Data Fusion: from Primary Metrology to Process Measurement. Proceedings of the $16^{\text {th }}$ IEEE IMTC. Venice, 3, 1325-1329.

[3] Sroka, R. (2004). Data Fusion Applied to the Minimization of Estimation Uncertainty. Metrology and Measurement Systems, 11(2), 107-122.

[4] Sroka, R. (2004). Data Fusion Methods Based on Fuzzy Measures in Vehicle Classification Process. Proceedings of the $21^{\text {th }}$ IEEE Instrumentation and Measurement Technology Conference. Italy. Como, 3, 2234-2239.

[5] Sroka, R. (2005). Data Fusion in Phase Angle Measurement System. Proceedings of $14^{\text {th }}$ IMEKO TC4 Symposium. Gdynia-Jurata, 1, 165-170.

[6] Gajda, J., Sroka, R., Żegleń, T. (2007). Accuracy analysis of WIM systems calibrated using pre-weighed vehicles method. Metrology and Measurement Systems, 14(4), 517-527.

[7] Burnos, P., Gajda, J., Piwowar, P., Sroka, R., Stencel, M., Żegleń, T. (2007). Accurate Weighing of Moving Vehicles. Metrology and Measurement Systems, 14(4), 508-516.

[8] Gajda, J., Piwowar, P. (2009). Identification of the human respiratory system during experiment with negative pressure impulse excitation. Metrology and Measurement Systems, 16(4), 569-583.

[9] Katulski, R.J., Namieśnik, J., Stefański, J., Sadowski, J., Wardencki, W., Szymańska, K. (2009). Mobile monitoring system for gaseous air pollution. Metrology and Measurement Systems, 16(4), 677-683. 
[10] Janiczek, J. (2009). Multiparameter approximation of transducer transfer function by kriging method. Metrology and Measurement Systems, 16(3), 479-491.

[11] Frączek, E., Mroczka, J. (2009). An accuracy analysis of small angle measurement using the Optical Vortex Interferometer. Metrology and Measurement Systems, 16(2), 249-258.

[12] Saltelli, A., Chan, K., Scott, E.M. (2000). Sensitivity Analysis. John Willey \& Sons LTD. New York.

[13] Szyper, M. (1998). Lipschitz's Measures of Measuring Systems Sensitivity to Variability of Parameters. SAMS, 30, 45-55.

[14] Szyper, M. (1995). Linear Parametric Modulation of a Phase Angle with Wide Range Deviation in Measurement Systems. Measurement. Elsevier Science, 16, 31-35.

[15] Szyper, M., Zielinski, T.P., Sroka, R. (1992). Spectral Analysis of Nonstationary Signals with Wide Phase Modulation. IEEE Trans. on Instrumentation and Measurement, 41(6), 919-920.

[16] Forrester, J.W. (2001). An Introduction to Sensitivity Analysis. Massachusetts Institute of Technology, Educational Materials.

[17] Engelbreck, A., Cloete, I., Żurada, J. (1995) Determining the Significance of Input Parameters Using Sensitivity Analysis. From Natural to Artificial Neural Computations. IWANN. Malaga, 382-388.

[18] Cukier, R.I., Fortuin, C.M., Schuler, K.E., Petschek, A.G., Schaibly, J.H. (1973). Study of the Sensitivity of Coupled Reaction Systems to Uncertainties in Rate Coefficients. I Theory. J. Chem. Phys., 59, 38733978.

[19] Prokott, E. (1978). Modulation und Demodulation. Dr A. Huthig Verlag Heidelberg Meinz Basel. 